\title{
Lifelogging Memory Appliance for People with Episodic Memory Impairment
}

\author{
Matthew L. Lee \& Anind K. Dey \\ Human-Computer Interaction Institute, Carnegie Mellon \\ 5000 Forbes Avenue, Pittsburgh, PA 15213 USA \\ $\{$ mllee, anind $\} @$ cs.cmu.edu
}

\begin{abstract}
Lifelogging technologies have the potential to provide memory cues for people who struggle with episodic memory impairment (EMI). These memory cues enable the recollection of significant experiences, which is important for people with EMI to regain a sense of normalcy in their lives. However, lifelogging technologies often collect an overwhelmingly large amount of data to review. The best memory cues need to be extracted and presented in a way that best supports episodic recollection. We describe the design of a new lifelogging system that captures photos, ambient audio, and location information and leverages both automated content/context analysis and the expertise of family caregivers to facilitate the extraction and annotation of a salient summary consisting of good cues from the lifelog. The system presents the selected cues for review in a way that maximizes the opportunities for the person with EMI to think deeply about these cues to trigger memory recollection on his own without burdening the caregiver. We compare our system with another review system that requires the caregiver to repeatedly guide the review process. Our self-guided system resulted in better memory retention and imposed a smaller burden on the caregiver whereas the caregiver-guided approach provided more opportunities for caregiver interaction.
\end{abstract}

\section{Author Keywords}

Lifelogging, information overload, Alzheimer's disease, memory impairment, caregiver burden.

\section{ACM Classification Keywords}

H5.m. Information interfaces and presentation (e.g., HCI): Miscellaneous.

\section{INTRODUCTION}

Episodic memory impairment (EMI) is the main symptom associated with Alzheimer's disease, a common

Permission to make digital or hard copies of all or part of this work for personal or classroom use is granted without fee provided that copies are not made or distributed for profit or commercial advantage and that copies bear this notice and the full citation on the first page. To copy otherwise, or republish, to post on servers or to redistribute to lists, requires prior specific permission and/or a fee.

UbiComp'08, September 21-24, 2008, Seoul, Korea.

Copyright 2008 ACM 978-1-60558-136-1/08/09...\$5.00. neurodegenerative disease that affects over 26 million people worldwide, with this number expected to quadruple by 2050 [2]. Recent episodic memory supports our sense of self [4] by enabling us to mentally travel back in time and relive our pleasant experiences and to be socially and physically engaged in our lives. Recent episodic memory impairment dramatically changes the lives of individuals with the impairment. Individuals struggle with the need to constantly readjust their own expectations of what they can and can no longer do.

EMI can lead to a loss in autonomy and control in individuals' lives, resulting in feelings of uncertainty, irritation, and frustration as they attempt to compensate for their memory loss but repeatedly fail. They may withdraw from interacting socially with others to avoid appearing incompetent and even develop depression as a result [17]. They must rely on others for support and often must repetitively ask their family caregivers for information about current and recent events. The lives of their family caregivers are also dramatically changed as caregivers become overburdened with providing for both the cognitive and physical needs of their loved one and can themselves develop depression or burnout that leads to reduced quality of care [1]. Thus, individuals with EMI and their caregivers struggle for a sense of normalcy-how things were before the onset of the disease-in their lives [3, 4]. In other words, their ideal situation would be to turn back the clock and live in a time before the disease changed their lives, when their lives were "normal." Restoring normalcy in their now changed lives involves restoring the memory abilities and independence that the disease took away from them.

Ubiquitous lifelogging systems (e.g., [12], [13], [19]) use wearable or embedded sensor technologies such as cameras, audio recorders, location trackers, and physiological sensors to passively and automatically record a user's personal experiences. Lifelogging technologies allow people with EMI to automatically record, review, and thus regain an awareness of meaningful personal experiences in their lives to maintain their sense of self [13]. Sellen et al. [15] showed that episodic details from a visual "lifelog" can be presented to users as memory cues to assist them in remembering the details of the original experience. Other successful systems leverage other modalities such as the Audio Memory Prosthesis [19] that records audio from 
personal experiences and presents it as searchable and browsable text. The Personal Life Log system [18] uses a combination of location sensors, physiological sensors, and real-time voice annotation to identify potentially interesting scenes in a continuous video $\log$.

However, lifelogging technologies often automatically capture an overwhelmingly large amount of data that can be both tedious and difficult to review. In particular, people with EMI often have other cognitive impairments that make it extremely difficult to review and engage with vast amounts of content that most lifelogging systems generate [16]. To be most effective in supporting memory, lifelogging systems need to create a salient summary of the lifelog that provides the most important information to present to the user in an engaging way.

One successful system, the Microsoft SenseCam, leverages sensors to selectively capture information and also leverages the caregiver to further select the important information [13]. The system uses a wearable digital camera that automatically takes photos triggered by onboard sensors. It was found to be helpful for assisting an individual with EMI from traumatic brain injury in remembering the details of personal experiences. The SenseCam system, despite its "smart" capture technique, still imposes a somewhat large amount of extra work on already overburdened caregivers by requiring them to look through all the captured photos, select the most important photos as cues, and repeatedly review the photos with the person with EMI. The additional work imposed on caregivers exacerbates the lack of normalcy in their lives as they have less time to take care of themselves.

Recent findings by Lee \& Dey [14] enable the development of content and context analysis techniques that can simplify the caregiver's task of filtering lifelog data. They explored what types of cues are the best for helping an individual to mentally relive an experience. A good cue serves as an anchor into the experience by which an individual can retrieve and recollect many vivid episodic details associated with that cue. They found that the best cues for an experience are determined by the type of the experience (such as people-based, location-based, action-based, or object-based experiences). Lifelogging systems can be designed to automatically filter the data they collect using simple heuristics based on the type of experience such as selecting instances of people, faces, or conversations in people-based experiences to present as memory cues.

While automatic filtering may be effective for significantly reducing the amount of information to review, the best cues within these categories for triggering recollection must also be either personally significant or distinctive [14]. Unfortunately, there are no simple heuristics to determine which content from the lifelog is most significant or distinctive. Thus, the caregiver must still be involved to select the significant and distinctive cues from the filtered content.
Furthermore, selecting the best cues is only half the process of supporting recollection for people with EMI. The second half is presenting the cues in a way so that people with EMI are able to recognize and process each cue deeply enough to feel like they are mentally reliving the experience (an important aspect of memory that makes individuals feel more normal) as opposed to passively learning previously forgotten details.

In this paper, we will describe the design of a lifelogging system building on the Microsoft SenseCam system [13] that passively captures people's experiences. Unlike the traditional SenseCam system, our system uses a hybrid cue selection strategy combining automated approaches with the expertise of the caregiver to select the most appropriate content from the lifelog to present as memory cues. Our system also provides a memory-stimulating, self-guided interaction for the person with EMI to recollect their experiences without repetitively burdening their caregiver during review. We will describe a comparative evaluation of our system that uses this self-guided review approach and the traditional Microsoft SenseCam system, which uses a caregiver-guided interaction to review lifelog data. We demonstrate that our design is better at restoring certain aspects of normalcy in the lives of people with EMI and their caregivers by better supporting the memory abilities of people with EMI and also by imposing less of a burden on caregivers. In the following section, we describe how our system captures, selects, and presents lifelogging data to aid episodic recollection for people with EMI.

\section{SYSTEM DESIGN}

We designed a lifelogging system that follows a three-step process of capture, cue selection, and review to support recollection of episodic memories (Figure 1). Using a combination of sensors, the system passively records experiences of the user's choosing. Then, the caregiver uses the CueChooser application to construct a narrative of the experience by selecting and annotating the most salient memory cues from the recorded lifelog data, with the help from content and context analysis, which is automated from the caregiver's perspective but, for the sake of our formative evaluation study, is currently performed via Wizard-of-Oz. Finally, with the MemExerciser application, the caregiver-chosen cues are presented to the person with EMI in a way that maximizes the opportunities for them to think deeply about the cues to remember associated details on their own without requiring repeated live help from their caregiver. In the following sections, we describe the rationale behind our design decisions.

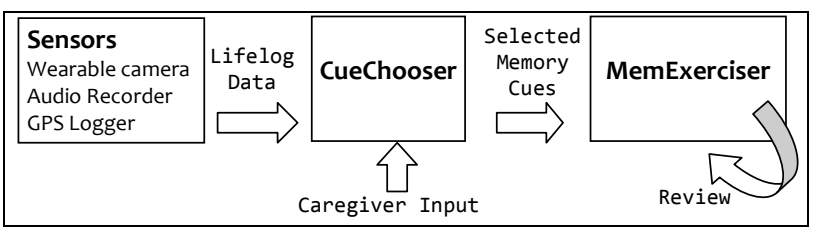

Figure 1. System Design: Capture, Selection, and Review 


\section{Automated Passive Capture}

Lifelogging technologies that use a passive capture approach automatically record the user's experience without the need for the user to consciously remember to initiate capture. The passive approach allows users to be less distracted by the technology itself but instead to focus their attention on participating in the experience [10]. A passive capture approach is even more appropriate for people who have difficulty remembering to initiate capture. In fact, many people in the early stages of Alzheimer's disease may not fully acknowledge the extent of their EMI and thus are often unwilling to take any additional effort to consciously record their experiences.

Our capture system records photographs, ambient voices and sounds, and location information from the user's experiences using three devices: the Microsoft SenseCam, an off-the-shelf voice recorder, and a GPS logger. The SenseCam [13] is a wearable digital camera worn at chest level that automatically takes photos. The SenseCam can use various sensors such as a three-axis accelerometer, temperature, light, and infrared sensors to determine "good" opportunities to take a photo. In our evaluation, we did not use sensor-triggered capture but rather set the SenseCam to take a photo every 30 seconds to ensure consistency. In addition to photos, other cues for memory include ambient voices and sounds [12, 19] and location information [14]. Ambient voices and sounds from the user's experience were recorded using an Olympus WS-310M voice recorder, placed in the user's shirt top pocket. GPS location information was also recorded every 1 to 5 seconds using a Wintec WBT-201 GPS logger. Users switched on all three devices together at the beginning of an experience and left them on until the end of the experience. Our system can record a vast amount of data that could potentially be used as cues to recollect an experience. However, it is infeasible for a person with cognitive impairment to review all these data, and therefore the next step in our approach involves filtering the lifelogging data for the best memory cues.

\section{Hybrid Cue Selection}

Our lifelogging system, despite only spanning three primary modalities (visual, audio, and location), can still capture an overwhelming amount of data from an experience. For example, if the system were used for an experience lasting eight hours, it would capture nearly 1000 photos, 480 minutes of audio, and 10,000 GPS waypoints. It is difficult for users (let alone users with EMI) to review all these data. Only the most relevant information that triggers a vivid episodic recollection of the experience should be extracted as a salient summary and reviewed as cues to mentally relive and reflect on the experience. Our approach leverages automated analysis and extraction of potentially helpful memory cues from the lifelog to filter it down to a more manageable size and then the expertise of the caregiver of the person with EMI to hand select meaningful memory cues from the filtered content to present to the person with EMI.

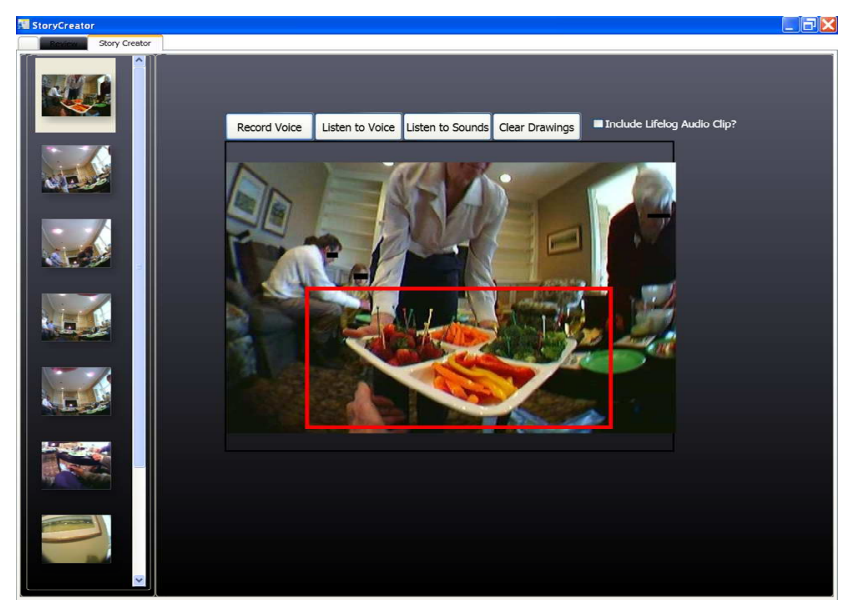

Figure 2 CueChooser user interface. The caregiver can view system-suggested cues in constructing a narrative, and provide visual and audio annotations to selected cues.

We have designed CueChooser (Figure 2), a computer application that uses automated heuristic-based content and context analysis to identify photos and sounds that might serve as good memory cues to construct in a cueing narrative for use by the person with EMI. Previous research [14] has found that people's experiences can be categorized as being people-based, location-based, action-based, and object-based, and these experiences can often be characterized by one or two types of cues. For example, memories of people-based experiences such as weddings and family get-togethers are best triggered by the people encountered during these experiences. Knowing the type of experience can help determine what data should be extracted for review. The user can tell the system the type of the experience so that it can apply the appropriate heuristics. For people-based experiences, our system identifies photos containing faces and suggests these as potentially good memory cues. Likewise for location-based experiences, the system analyzes the GPS $\log$ to identify situations where the user is entering, dwelling in, or leaving a particular location. For object-based experiences, the system can use the SenseCam accelerometer or GPS data to look for instances where the user is remaining relatively stationary, as this may indicate that the user is looking at something interesting. For action-based experiences, image set summarization and segmentation techniques [7] are used to break up the experience into distinct scenes and representative images are selected from these segments to represent the experience.

Good memory cues should be recognizable, distinctive, and personally significant [14]. Automated heuristics to detect these characteristics are still lacking. Humans are better at identifying these characteristics, and in particular, family caregivers of people with EMIs regularly select cues that fit these criteria [14], when helping their loved one recollect a past experience. Hence our system allows the caregiver to make the final selection of lifelog data to be used as memory cues. After the caregiver specifies the type of experience and CueChooser filters the lifelog data according to heuristics appropriate to the type of experience 
as described above, the caregiver can use the suggested lifelog photos and associated recorded audio as interesting points in the lifelog to browse. The caregiver can choose a combination of suggested cues and other lifelog data to include in a cueing narrative for the person with EMI. The caregiver can record a voice annotation to describe the photos and captured audio and to provide personallysignificant details to aid recollection just as they do when reviewing a photo album together with the person with EMI. The caregiver can also highlight portions of photographs by sketching boxes to draw the viewer's attention to particularly meaningful details. Using CueChooser, caregivers can construct and annotate a narrative containing meaningful cues from the lifelog with the help of automated content and context analysis.

\section{Progressive Revealing of Cues}

Caregivers often support the episodic memory of their loved ones using a cueing process where they use particular details of an experience to help the care recipient recollect other details of the experience. People with EMI enjoy "Aha!" moments, when a particularly good cue triggers their own recollection of rich details of the experience [14]. However, overburdened caregivers sometimes do not complete the cueing process because they do not have the time or grow tired of repetitively engaging in a cueing process for the same experience. Existing lifelogging systems such as MyLifeBits [10] and LifeStream [8] do not readily present their data in a supportive cueing process but rather rely on the user to search for items.

Hawkey et al. [11] introduced the concept of an information appliance, a device with the sole purpose of allowing a person with Alzheimer's disease to find the answers to the questions they most frequently ask their caregivers. We follow this approach in designing our MemExerciser system as a standalone memory appliance that people with EMI can use to refresh and exercise their memory of recent experiences in their lives. Our MemExerciser review system engages the person with EMI in a cueing process where additional cues are revealed progressively to maximize opportunities to recollect associated details without repeated help from a caregiver. This can increase the independence of the person with EMI and also minimize the extra burden on the caregiver caused by having to repetitively engage in a cueing process.

MemExerciser consists of an application (Figure 3) installed on a Toshiba M200 tablet PC so that it mimics a picture frame that users can pick up and use without having to worry about operating a computer or bothering their caregiver. It has the appearance of a specialized appliance as opposed to a general purpose computer because the MemExerciser is the only application visible to the user. In MemExerciser, the visual and audio cues chosen by the caregiver are presented in a slideshow narrative. At the beginning of the slideshow the caregiver-specified title, date, and description of the experience are displayed to

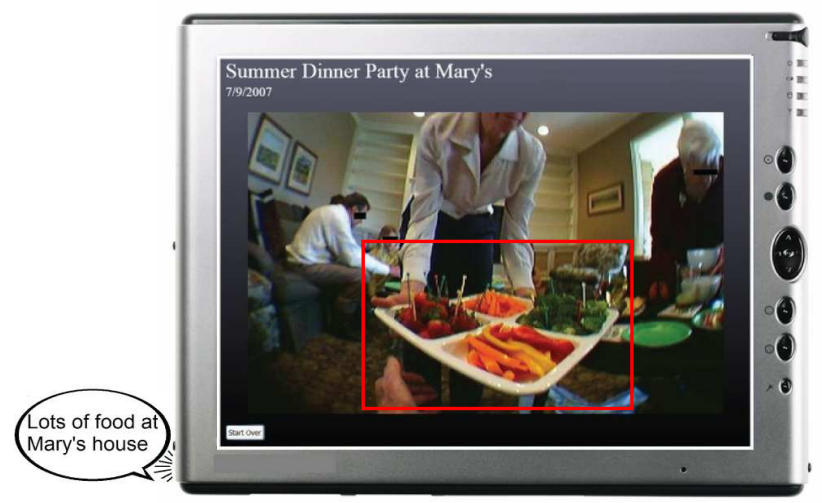

Figure 3. MemExerciser user interface: tapping on the screen displays pictures, and plays back lifelog audio and caregiver's voice annotation.

orient the user. The user can tap the tablet with the stylus to show the first photo. For each photo in the slideshow, cues are revealed progressively to give the user multiple opportunities to think deeply about each cue, exercise his memory processes, and recollect associated episodic details on his own. The user has an opportunity to peruse the photo and think deeply about it to recognize it and to recollect other details from the experience. Tapping the screen again, the clip of the lifelog audio recorded when the photo was taken is played back. Listening to the audio playback, the user has another opportunity to recollect other details of the experience using this additional cue. When the user taps the screen again, MemExerciser plays back the caregiver's voice annotation while displaying any visual annotations created by the caregiver to highlight portions of the photo. Again, the user has another opportunity to use the additional information to mentally relive the original experience. Tapping the screen again takes the user to the next photo, where the same pattern of progressive revealing of additional cues is repeated. The MemExerciser system follows a similar cueing process used by the caregiver in the absence of any technological support but has the benefit of being much more patient than the caregiver. The user with EMI can review cues at a more comfortable pace with less pressure to "perform" quickly for the caregiver.

\section{EVALUATION}

The main approach of our lifelogging system includes preselecting cues from lifelog data based on input from the caregiver who is assisted by automated content and context analysis (with the CueChooser application) and progressively revealing cues to facilitate active selfrecollection (with the MemExerciser application). We compared our system's "Self-Guided" approach with two other approaches that required different contributions from the caregiver: "Caregiver-Guided" and "CaregiverNoFilter," The current gold standard "Caregiver-Guided" approach [13] requires the caregiver to filter through the entire set of captured images to select appropriate cues and to repeatedly guide the person with EMI through the selected cues. The Caregiver-NoFilter approach is similar to this approach without the extra step of filtering and cue 
selection. In our evaluation, we measured how effective each approach was for restoring aspects of normalcy in participants' and caregivers' lives, by supporting memory recollection and confidence and by reducing the amount of caregiver burden imposed by the system. We conducted a repeated measures within-subjects study to test the following hypotheses:

Hypothesis 1: Participants will be able to recall and recollect more of the details of their experiences using the Self-Guided approach than using the Caregiver-Guided or Caregiver-NoFilter approaches.

Hypothesis 2: Participants will feel more confident about their memory when using the Self-Guided approach than using the Caregiver-Guided or Caregiver-NoFilter approaches.

Hypothesis 3: The Self-Guided approach will impose less additional burden on the caregiver than the CaregiverGuided or Caregiver-NoFilter approaches.

We recruited three participants and their caregivers (Table 1) from the local Alzheimer's Association support group and a local retirement community. Two participants (P2 \& P3) were diagnosed with Mild Cognitive Impairment (MCI), which is a precursor condition to Alzheimer's disease where people experience recent episodic memory impairment (EMI) but do not have the executive or language problems of the later stages of Alzheimer's. One participant (P1) was diagnosed with general EMI. All participants had significant difficulty remembering the details of recent experiences a couple of days after an experience, in the absence of memory aids.

\begin{tabular}{lllll}
\hline Dyads & Sex & Age & Relationship & Condition \\
\hline P1/CG1 & M/F & $79 / 82$ & husband/wife & EMI \\
P2/CG2 & M/F & $76 / 80$ & husband/wife & MCI \\
P3/CG3 & M/F & $77 / 79$ & husband/wife & MCI \\
\hline
\end{tabular}

Table 1. Characteristics of participants $(P)$ and their caregivers (CG) and the participant's memory condition. Episodic Memory Impairment (EMI) and Mild Cognitive Impairment (MCI).

We used a within-subjects design to control for individual differences in memory functioning and caregiver relationship. Each couple participated in all four experimental conditions: Control (no intervention), SelfGuided, Caregiver-NoFilter, and Caregiver-Guided. We varied the experimental conditions to avoid an order effect and our analysis showed no resulting order effects.

Each experimental condition lasted four weeks and began with the participants going on a personally significant experience (e.g., a museum visit, a trip out of town, a dinner party, etc.) while wearing our lifelogging capture system consisting of the SenseCam, audio recorder, and GPS logger. For each experience, caregivers and participants processed and reviewed the lifelog data using one of four different approaches corresponding to the four experimental conditions described below.
Each experimental condition began with participants turning on the lifelogging system, going on a one-day experience, and turning it off immediately afterwards. On the day following the experience (referred to as Dayl of the experimental condition), the participant's memory is assessed using a free recall task where an experimenter asks the participant to recall as many details as he can about the experience. During each experimental condition's first two weeks (referred to as the "intervention period") on Day1, Day3, Day5, Day8, Day10, Day13, participants used either no intervention (the Control condition) or reviewed the recorded lifelogging data according to one of three review approaches: Self-Guided, Caregiver-Guided, or CaregiverNoFilter. We were careful not to overlap the intervention periods between conditions. At the end of the two-week intervention period, on Day14, and two weeks after that, on Day28, the participant's memory is again assessed using free recall. We verified the participant's memory of the experience using the account provided by the caregiver as ground truth.

On Day1 of the Self-Guided condition, caregivers used the CueChooser application to select cues from the lifelog data, annotate them, and construct a narrative. As described earlier, we used a Wizard-of-Oz technique to manually simulate the "automatic" selection of appropriate photos to suggest as good cues for the caregiver to include in the narrative. For P1's dinner party, a people-based experience, we manually looked at the photos to find large faces with strong features and good illumination that an average face detection software would be able to detect. For P2's visit to an indoor art exhibit, an object-based experience, we examined the accelerometer data to find moments of high and low movement as proxies for interesting exhibits. We took roughly $20 \%$ of the photos corresponding to the highest and lowest motion peaks. For P3's trip to an outdoor zoo, an object-based experience, we examined the GPS $\log$ to find moments when the participant was moving slowly or staying in one place for more than two minutes as indicators of when he was looking at something interesting. Caregivers could choose to include these suggested picture cues in the narrative or not. The participants then reviewed the caregiver-constructed narrative of cues using the MemExerciser tablet-based application on their own without requiring the caregiver to repetitively guide them through the cues. Caregivers did not engage the participants in a cueing dialog about the experience in this condition but instead only prompted the participants to use MemExerciser to refresh their memory on the appropriate days of the intervention period.

In the Caregiver-Guided condition, our participants followed a review procedure similar to the previous study [13] with SenseCam images and memory. On Day1, the caregivers looked through all the pictures and selected a subset to use in a discussion about the experience with the participant. On each review day, participants and caregivers sat down together to review only photos using the 
SenseCam Picture Viewer according to the same review schedule as in the Self-Guided condition. This viewer allowed caregivers to playback the photos one at a time or as a flipbook movie using rapid serial visual presentation. In contrast to the Self-Guided condition where the participant reviewed a caregiver-constructed annotated narrative on their own, the Caregiver-Guided condition requires the caregiver to be present to guide the review process by walking through the photos, describing them, and engaging the participant in a dialog with questions about the experience.

The Caregiver-NoFilter condition was identical to the Caregiver-Guided condition except the caregivers did not take the extra step to select out a subset of photos but instead walked the participant through all the photos taken by the SenseCam, skipping over any at their discretion.

In the Control condition, participants also went on a personally significant experience but they did not use any intervention to help them review the experience. Their memory for that experience was also tested using an identical schedule as in the other conditions.

\section{Measures}

For each experimental condition, we measured the participant's ability to freely recall the details of the experience three times: immediately after the experience (Day1), after the intervention period (Day14), and four weeks after the experience (Day28). Participants classified the vividness of each detail they recalled using the Remember-Know-Guess scale [9]. Participants classified a detail as "remembered" if they could recall vivid episodic phenomena such as imagery, feelings, sounds, and locations associated with that detail. Participants classified details they recalled as "known" if they were certain the detail actually happened but could not retrieve any specific episodic phenomena about it. They classified details as "guessed" if they were unsure about whether they were recalling correctly. P1 had difficulty in making such distinctions, so we analyzed a transcript of his free recall session for specific episodic details like feelings and imagery, key features of "remembering" versus "knowing."

As a measure of how the approaches affected the participants' confidence in their own memory, participants completed a version of the Metamemory in Adulthood (MIA) questionnaire [6], shortened from 100+ items to 20 items to focus specifically on their ability to recall episodic memories. Participants completed the MIA questionnaire at the very beginning of the study (as a baseline) and after each experimental condition.

For caregiver burden, we measured the amount of time caregivers spent using the interventions applied in the experimental conditions. After completing all conditions, caregivers were interviewed separately where we asked them which approach, Self-Guided, Caregiver-Guided, or Caregiver-NoFilter, they would prefer to use and why.

\section{RESULTS}

Each participant/caregiver dyad went on four different experiences and completed all four experimental conditions, except P1/CG1 who did not participate in the Caregiver-Guided condition. Even though they found the various interventions interesting, the caregiver decided that she did not want the extra complications of another round to take up time in her busy life. For our statistical analysis, we followed a standard procedure for estimating P1's missing value for a two-way ANOVA: the least-squares estimate, which is based on both the means for each condition and for each participant.

In the Self-Guided condition, caregivers were able to look through the lifelog data, make decisions about the suggested media, and select and annotate data to use as cues. All participants were able to successfully use the MemExerciser tablet-based system on their own with after being prompted by their caregivers. Based on the memory recall scores, metamemory assessments, and time data, we now consider whether they supported our hypotheses:

\section{Hypothesis 1: Memory Recall}

At the conclusion of each experimental condition, participants recounted the details of the original experience and rated the vividness of each recounted detail using the Remember-Know-Guess scale. From these data, we consider two operationalized measures for how well participants were able to recall the breadth and depth of details from their experiences: the total number of details recalled and the percentage of "remembered" details.

\section{Total Number of Details}

As an indicator of how well the interventions supported the participant's episodic memory, we looked at the total number of details recounted correctly, as verified by the caregiver. As shown by the negative slopes in all conditions in Figure 4, participants forgot details of the experience as more days elapsed after the original experience. Using a non-parametric statistical test, a Friedman repeatedmeasures ANOVA on ranks, we found there were no significant differences in the rate of forgetting at Day14. However, when looking at the longer-term recall scores at Day28, we find marginal differences that approach

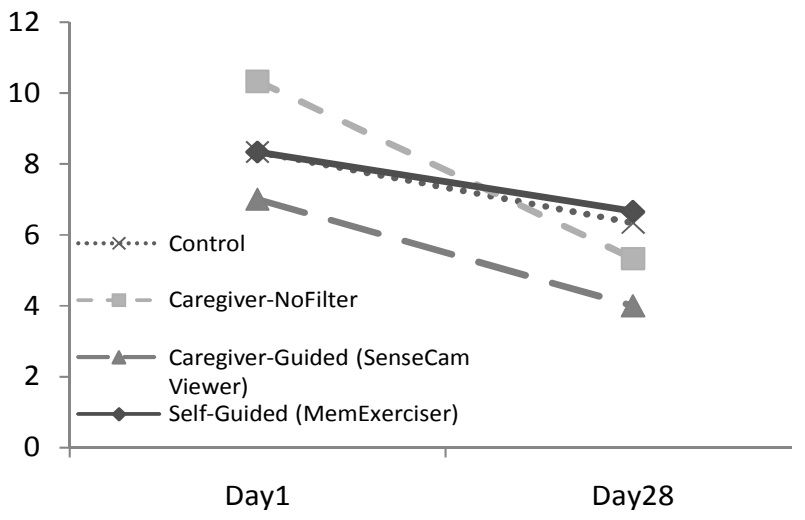

Figure 4. Mean Number of Details Recalled 
significance $(\mathrm{p}<0.07)$ among the experimental conditions in the rate of forgetting (shown as the slopes of the lines in Figure 4). This suggests that the Caregiver-Guided and the Caregiver-NoFilter conditions resulted in more rapid forgetting than the Self-Guided condition. One limitation in our study design is that some experiences have different number of details to recall. Moreover, some experiences may be inherently more interesting and easier to recall, which may be the reason for the relatively low forgetting rate in the Control condition in Figure 4. To minimize this impact, we made sure each experience was one the individual with EMI would want to reflect on afterwards. These data provide marginal support Hypothesis 1 and suggest that the MemExerciser's Self-Guided approach resulted in participants forgetting fewer details than the Caregiver-Guided approach. With the ability to remember more details, our participants' memory exhibited greater breadth of the experience. Our next measures examine the depth of their memory for the experience by looking at vividness of the recalled details.

\section{Percentage of Remembered Details}

We measured the vividness of our participants' memories by looking at the percentage of details they judged as remembered on the Remember-Know-Guess scale of vividness [7]. The higher the percentage of remembered details for an experience, the more likely the participant would feel as if he were mentally reliving the experience using his memory rather than simply knowing the abstract facts about the experience.

To account for the differences in the initial vividness (percentage of details judged as remembered) of each experience, we analyzed the change in vividness over time (shown as the slopes of the lines in Figure 5) rather than the absolute percentages. We found statistically significant differences among our experimental conditions at Day14 (Figure 5) using a Friedman repeated-measures ANOVA on ranks $(\mathrm{p}<0.05)$, with the Self-Guided condition resulting in an average increase in remembered details after the twoweek intervention period versus an average decrease in remembered details in the other conditions. A similar

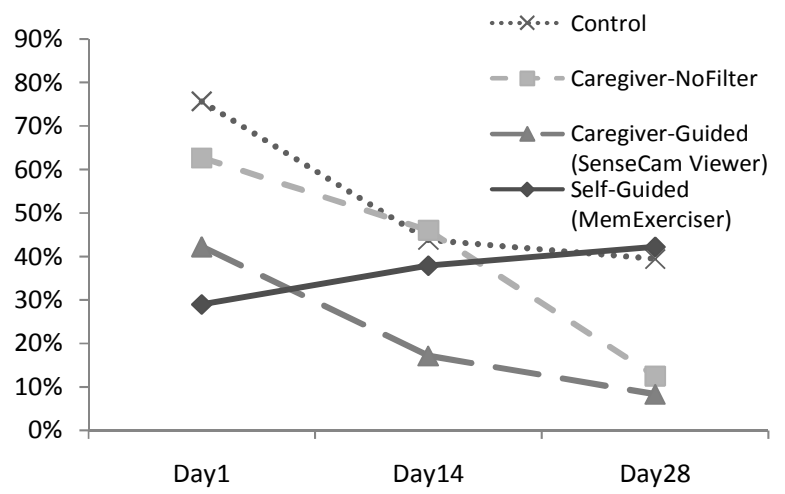

Figure 5. Percentage of Details Judged as Remembered. The Self-Guided condition increased in remembered details. analysis for the change in vividness over four weeks, reveals a similar trend $(\mathrm{p}<0.05)$ where the Self-Guided condition increased and maintained a level of remembered details (and thus the feeling of reliving the experience) whereas the other conditions decreased in vividness. The increase in the percentage of remembered details for the Self-Guided condition provides support for Hypothesis 1 .

\section{Hypothesis 2: Memory Confidence}

Participants completed a customized version of the Metamemory in Adulthood (MIA) questionnaire at the very beginning of the study (for a baseline measurement) and after each experimental condition. We used a metamemory assessment taken at the beginning of the study as a baseline instead of during the Control condition because we did not want participant's use of the experimental interventions to unduly influence their baseline beliefs. We included four scales in the MIA questionnaire to assess various aspects of their own beliefs about their memory: capacity, anxiety, locus (control), and achievement (the desire to maintain or improve memory). Summing these scales together for an overall measure for self-assessed confidence in memory, we observed that participants were most confident about their memory after the Self-Guided condition (Mean=63.3, $\mathrm{SD}=6.7$ ), followed by the Caregiver-Guided condition (Mean=59.6, SD=4.7), then by the Caregiver-NoFilter (Mean=59.0, $\mathrm{SD}=4.0$ ) and least confident at the Baseline assessment (Mean=54.3, SD=9.6) (Figure 7). A Friedman repeated-measures ANOVA on ranks $(\mathrm{p}=<0.05)$ revealed that participants felt significantly more confident about their memory after the Self-Guided condition than in the Baseline level, whereas the Caregiver-Guided and Caregiver-NoFilter conditions were not significantly different from Baseline. These data provide support for a modified form of Hypothesis 2: the Self-Guided approach can help the participant feel more capable and confident in their memory abilities than normal whereas the CaregiverGuided and Caregiver-NoFilter approaches do not.

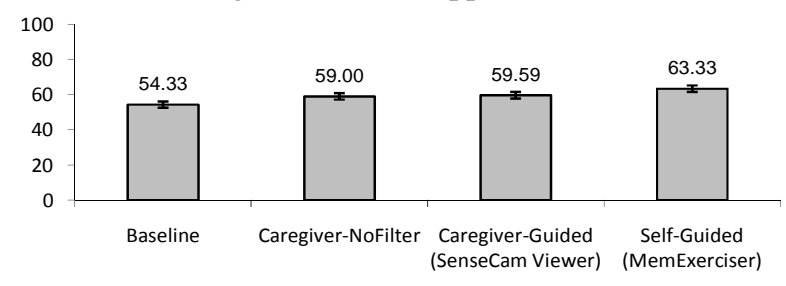

Figure 6. Participant's self-assessed memory confidence using the Metamemory in Adulthood Questionnaire

Hypothesis 3: Caregiver Burden

In addition to the improved memory outcomes for the person with memory impairment, we also looked at the amount of extra work imposed by each review method on the caregiver. Caregivers are physically and mentally overburdened and seek ways to minimize the amount of extra work they have to do. 


\begin{tabular}{|c|c|c|c|}
\hline & Self-Guided & $\begin{array}{l}\text { Caregiver-Guided } \\
\text { filter + review }=\text { total }\end{array}$ & Caregiver-NoFilter \\
\hline CG1 & $67 \mathrm{mins}$ & $\mathrm{n} / \mathrm{a}$ & $120 \mathrm{mins}$ \\
\hline CG2 & $64 \mathrm{mins}$ & $19+52=71 \mathrm{mins}$ & $86 \mathrm{mins}$ \\
\hline CG3 & $55 \mathrm{mins}$ & $12+54=66 \mathrm{mins}$ & $102 \mathrm{mins}$ \\
\hline
\end{tabular}

Table 2. Amount of time required by each caregiver (CG) to use each review method. The Self-Guided review method required the least amount of the caregiver's time.

With the Self-Guided approach, caregivers spent all their time at the beginning to select cues, annotate them, and create a slideshow narrative of the experience. In the Caregiver-Guided and Caregiver-NoFilter conditions, caregivers spent most of their time in review sessions with the person with memory impairment spread out over the two week intervention period. Table 2 shows the SelfGuided approach had a greater upfront cost in time but still demanded the least overall amount of the caregiver's time. Spending time initially filtering the lifelog in the CaregiverGuided condition also resulted in a lower overall time burden when compared to the Caregiver-NoFilter condition.

\section{DISCUSSION}

Our evaluation study looked at how different interaction designs (Self-Guided, Caregiver-Guided, and CaregiverNoFilter) for a lifelogging system could help restore two aspects of normalcy in the lives of individuals with EMI and their caregivers: 1) the ability and confidence of the individual with EMI to recollect recent experiences and 2) relieving the caregiver of the burden of repetitively supporting the individual's memory.

The MemExerciser's Self-Guided review approach was the most effective at restoring a sense of normalcy for the individual with EMI through supporting his ability to recollect recent experiences. Participants were able to recall more details of the original experience when using the SelfGuided approach (when compared with the CaregiverGuided and Caregiver-NoFilter approaches) because they were able to review at a more comfortable pace and think more deeply about each cue. The Self-Guided approach progressively revealed more details to support deeper processing of memories which can make them easier to recall [5]. In our interviews, P2 said that when using the Self-Guided approach, he "would be more inclined to take more time and look for more details... and get more out of it...because you're looking for things you don't normally see [in the Caregiver-Guided approach]." His caregiver, CG2 said, "It helps him focus his thoughts, plus [the ambient] voices for the slides makes it easier for him to recall each picture." The Self-Guided approach enabled participants to retrieve additional details that they did not initially recall, which produced richer, "more lifelike" (P3) accounts as measured by the increase in percentage of remembered details over time.

In addition to the actual ability to recall recent episodes, feeling confident in the ability to recall is another important aspect of feeling normal. Based on responses to the Metamemory in Adulthood questionnaire [6], our study showed that participants felt the most confident about their memory abilities when using the Self-Guided approach to assist them to remember recent experiences. Qualitative feedback from our participants corroborated our quantitative results. P1 said that reviewing a narrative on his own "refreshed his recollection" of the original experience. P2 said, "It brings back memories that I wouldn't normally have remembered...It's a remarkable device." The Self-Guided approach helped our participants feel more normal in both their ability to recall recent experiences and their confidence in their memory abilities.

The value of normalcy also extends to the caregiver. The caregiver values her own independence from the person with EMI, which requires the person with EMI to be independent himself. The caregiver therefore strives to minimize the amount of extra work she needs to do in providing care. As with any intervention, reviewing lifelog cues to support memory imposes extra work on the caregiver. From our results, the Self-Guided approach, in which the caregiver authors a slideshow narrative only once instead of repetitively going through the lifelog data, required the least amount of time, when compared to the Caregiver-Guided and Caregiver-NoFilter approaches. Moreover, should participants continue to use these approaches to reinforce their memory, the time burden on the caregiver would continue to grow with the CaregiverGuided and Caregiver-NoFilter approaches whereas it would not increase with the Self-Guided approach. In our interviews, all caregivers said that the Self-Guided approach required the least amount of work. CG1 said the Caregiver-Guided approach required more work "because I had to be involved [during the reviewing]." CG2 said "I had to go through it with [P2] every time instead of him just doing it himself." In fact, when we asked CG2 which review method she would most like to use, she chose the Self-Guided approach and laughingly described herself as being "lazy" because she would rather do something else (such as taking care of her many household chores or reading her novels) instead of going through the same pictures again and again with her husband. CG3 said a drawback with the Caregiver-Guided approach was that she did not like reviewing the lifelog with her husband if she was not in the mood that day. CG2 and CG3 desired to restore a state of normalcy where they do not need to repetitively attend to their husband as a caregiver but rather where they can attend to their own well-being. The SelfGuided approach allows the person with EMI to repetitively refresh his memory of recent experiences on his own which minimizes the additional amount of caregiving work required of the caregivers.

However, CG1's preferences revealed other factors that contribute to a sense of normalcy. CG1 preferred the Caregiver-Guided approach because she enjoyed reviewing the photos with her husband as a shared activity. She said, 
"[The Caregiver-Guided approach] was something we did together - which doesn't happen often." EMI often makes it difficult for individuals to have meaningful conversations because they lack memories of recent experiences to talk about. The Caregiver-Guided review approach enabled CG1 to engage her husband actively in a conversation about a recent shared experience. We observed CG1 behaving noticeably more affectionately toward her husband after spending 30 minutes sitting on the couch with him to guide him through the lifelog data. For CG1, the ability to have a meaningful conversation with her husband is an important part of feeling normal. CG1 also preferred the CaregiverGuided approach because she felt her husband lacked the motivation to review the lifelog data on his own. Instead, she felt that by going through the lifelog data together with him, she played a greater role in helping him exercise his brain, slow the rate of cognitive decline, and even potentially restore some memory function that he had lost. CG1 said of P1, "[The Caregiver-Guided approach] helps him use his memory...it helps him use that part of his brain that he wouldn't use otherwise. That's good." For CG1, another important part of restoring normalcy in her life was helping her husband restore his declining memory abilities to a more normal, more competent state. Taking an active role in exercising her husband's memory was one way she was trying to "turn back the clock" on the cognitive decline.

MemExerciser's Self-Guided approach had three main differences from the Caregiver-Guided approach: types of sensing, hybrid vs. caregiver-only filtering, and self $v s$. caregiver review. These two different approaches were successful at restoring different aspects of normalcy for individuals with EMI and their caregivers. The Self-Guided approach with its additional ambient audio sensing and its review method that progressively revealed cues was more effective at restoring a sense of normalcy for the person with EMI by improving their ability to recall and feel confident about recent experiences. The Self-Guided approach's computer-assisted filtering and one-time narrative construction helped restore a sense of normalcy for caregivers by minimizing additional work imposed on them. On the other hand, the increased conversational interactions and opportunities to play an active role in slowing cognitive decline afforded in the Caregiver-Guided approach help caregivers (those who value conversation and an active role over minimizing the burden of caregiving) restored a sense of normalcy in their lives.

Our evaluation also gave us an opportunity to better understand how older adults would integrate lifelogging technologies in their lives. Our participants expressed an interest in logging only significant and meaningful experiences rather than everyday routines. Caregivers frequently were apprehensive about whether they correctly switched on/off the various capture devices and whether they actually recorded anything during the experience. Integrating all the functionality into one device with one physical switch could help reduce the caregiver's concerns about "getting it right" when switching it on/off. However, the capture devices did not impact the enjoyment of their outings. In fact, in most cases, participants usually forgot they were recording their experiences.

For the hybrid cue selection task, caregivers used many of the suggested photos but were also curious about what other photos and sounds were recorded. They frequently used the suggested photos to explore other content captured before or after the suggested photos. Our caregivers occasionally employed visual search through large portions of content to find a particular scene to include in the narrative. Tools to support the cue selection process should allow for both high-level access to the content as well as the ability to drill into the content details to find a particular item of interest.

Our participants had varying levels of interest in the cue review activity. P1 had little interest in reviewing what happened recently and preferred to read rather than look at photos on his own. Likewise, P2 preferred to nap and P3 preferred to watch television rather than to look at a slideshow of a recent event. These impressions indicate that the activity of reviewing recent life experiences may not be motivating enough by itself. Instead, reviewing of lifelog cues should be integrated with other activities (such as storytelling, photosharing, or scrapbooking) where reminiscing about recent experiences can be a healthy side effect rather than the main purpose. In fact, in one case, P3 was motivated to review the slideshow when his daughter came to visit and shared his trip to the zoo with her. Other factors that impacted the cue reviewing experiences were the visual and auditory limitations of our participants. Our participants may not have angled the tablet screen perfectly so that the low resolution photos would be displayed optimally. P1 had a hearing limitation that made the ambient lifelog audio difficult to comprehend. Designers of technology for people with EMI, many of whom are older adults, should remember to consider their visual and auditory limitations.

Our evaluation study has some limitations. The study only included three participants and their caregivers. Recruiting from this population is particularly difficult because caregivers are often overburdened. To make the most of our limited number of participants and to account for individual differences, we used a more powerful within-subjects study design. For our analysis, we used non-parametric statistics based on ranking rather than absolute values, which revealed some statistically significant trends that support our hypotheses about memory recall and confidence. The qualitative impressions of our participants also corroborate our quantitative findings. Nonetheless, further evaluations with more participants and experiences would improve the generalizability of our results. The study also relied only on participants' self-reported impressions about the cognitive processes such as the deeper processing of cues that were critical for supporting a more vivid recollection in the SelfGuided condition. More objective measures such as brain imaging may help better uncover these cognitive processes. 


\section{FUTURE WORK}

For our evaluation, we simulated the automatic cue suggestion with a Wizard-of-Oz technique to avoid spending time on implementation before validating our hybrid cue selection approach. We are currently integrating automated content and context analysis techniques (such as face detection, voice detection, and segmentation using movement and location, e.g., [7]) into our system to select cues appropriate for the type of experience. We will also explore ways to simplify the narrative authoring process to reduce the burden on caregivers. Details from a caregiverguided review session can be automatically extracted to create a narrative that the person with EMI can review on his own. We can use machine learning to learn which features of lifelog data are meaningful for each user to further automate the process of producing a salient cueing summary of the experience. We also will explore the use of fun and useful review interactions such as storytelling, scrapbooking, and games to motivate recollection.

\section{CONCLUSION}

We have designed and built a ubiquitous lifelogging system that captures photos, audio, and location information from a user's personal experiences to assist recollection for people with episodic memory impairment (EMI). Our system uses a hybrid cue selection strategy combining automated content filtering with the expertise of the family caregiver to create a salient summary of the captured data by selecting the most appropriate content from the lifelog to include in a narrative of memory cues. The MemExerciser application presents this narrative to the person with EMI with a Self-Guided approach that maximizes opportunities for them to recollect and exercise their memory on their own without requiring repeated help from the caregiver. The Self-Guided review method with its progressive revealing of cues was more effective at restoring a sense of normalcy for the person with EMI by improving their ability to recall and feel confident about recent experiences. The Self-Guided approach's computer-assisted filtering and one-time narrative construction also helped restore a sense of normalcy for caregivers by minimizing the amount of additional work imposed onto them. In contrast, the Caregiver-Guided approach does not support memory as well and imposes more of a burden on the caregiver, but it can restore other aspects of normalcy in caregiver's lives by giving them opportunities to have a conversation with their loved one and play an active role in slowing cognitive decline. Thus caregivers can benefit from using the SelfGuided approach when they feel more burdened and Caregiver-Guided approach when they want a more active role in caregiving.

\section{ACKNOWLEDGMENTS}

The authors would like to thank Microsoft Research for providing hardware and financial support, Sara Kiesler for helping with the statistics, and our study participants for their contributions. This work was supported by NSF grant DGE-0333420 and the Quality of Life Technology Center.

\section{REFERENCES}

1. Almbert, B., Grafstrom, M. and Winblad, B. (1997). Caring for a demented elderly person - burden and burnout among caregiving relatives. Journal of Advanced Nursing, 25 (1). 109-116.

2. American_Health_Assistance_Foundation. http://www.ahaf.org/alzdis/about/adabout.htm.

3. Butcher, H.K., Holkup, P.A. and Buckwalter, K.C. (2001). The Experience of Caring for a Family Member with Alzheimer's Disease. West J Nurs Res, 23 (1). 33-55.

4. Clare, L. (2003). Managing threats to self: awareness in early stage Alzheimer's disease. Social Science \& Medicine, 57 (6). 1017-1029.

5. Craik, F.I.M. and Tulving, E. (1975). Depth of processing and the retention of words in episodic memory. Journal of Experimental Psychology: General, 104 (3). 268-294.

6. Dixon, R. and Hultsch, D. (1983a). Metamemory and memory for text relationships in adulthood: A cross-validation study. Journal of Gerontology, 38. 689-694.

7. Doherty, A.R., Smeaton, A.F., Lee, K. and Ellis, D. (2007). Multimodal Segmentation of Lifelog Data. Proc. RIAO, Pittsburgh, PA, 2007.

8. Freeman, E. and Gelernter, D. (1996). Lifestreams: a storage model for personal data. ACM SIGMOD Record, 25 (1). 80-86.

9. Gardiner, J.M., Ramponi, C. and Richardson-Klavehn, A. (1998). Experiences of Remembering, Knowing, and Guessing. Consciousness and Cognition, 7 (1). 1-26.

10. Gemmell, J., Williams, L., Wood, K., Lueder, R. and Bell, G. (2004). Passive Capture and Ensuing Issues for a Personal Lifetime Store. Proc. CARPE'04. 48 - 55

11. Hawkey, K., Inkpen, K.M., Rockwood, K., McAllister, M. and Slonim, J. (2005). Requirements gathering with Alzheimer's patients and caregivers. Proc. ASSETS 2005. 142-149.

12. Hayes, G.R., Patel, S.N., Truong, K.N., Iachello, G., Kientz, J.A., Farmer, R. and Abowd, G.D. (2004). The Personal Audio Loop: Designing a Ubiquitous Audio-Based Memory Aid. Proc. Mobile HCI. 13-16.

13. Hodges, S., Williams, L., Berry, E., Izadi, S., Srinivasan, J., Butler, A., Smyth, G., Kapur, N. and Wood, K. (2006). SenseCam: a Retrospective Memory Aid. Proc. UBICOMP 2006. 81 - 90

14. Lee, M.L. and Dey, A.K. (2007). Providing Good Memory Cues for People with Episodic Memory Impairment. Proc. ASSETS 2007. 131 - 138

15. Sellen, A.J., Fogg, A., Aitken, M., Hodges, S., Rother, C. and Wood, K. (2007). Do life-logging technologies support memory for the past?: an experimental study using sensecam. Proc. CHI 2007. 81-90.

16. Silveri, M.C., Reali, G., Jenner, C. and Puopolo, M. (2007). Attention and Memory in the Preclinical Stage of Dementia. Journal of Geriatric Psychiatry and Neurology, 20 (2). 67-75.

17. Steeman, E., de, C., Dierckx, B., Godderis, J. and Grypdonck, M. (2006). Living with early-stage dementia: a review of qualitative studies. Journal of Advanced Nursing, 54. 722-738.

18. Tancharoen, D., Yamasaki, T. and Aizawa, K. (2005). Practical experience recording and indexing of Life Log video. Proc. CARPE 2006. 61-66.

19. Vemuri, S., Schmandt, C. and Bender, W. (2006). iRemember: a personal, long-term memory prosthesis. Proc. CARPE 2006. $65-74$. 